\title{
ALGEBRA STRUCTURES ON THE TWISTED EILENBERG-ZILBER THEOREM
}

\author{
V. Álvarez, J. A. Armario, M. D. Frau, and P. Real \\ Departamento de Matemática Aplicada I, Universidad de Sevilla, \\ Sevilla, Spain
}

Let $G, G^{\prime}$, and $G \times{ }_{\tau} G^{\prime}$ be three simplicial groups (not necessarily abelian) and $C_{N}(G) \otimes_{t} C_{N}\left(G^{\prime}\right)$ be the “twisted" tensor product associated to $C_{N}\left(G \times_{\tau} G^{\prime}\right)$ by the twisted Eilenberg-Zilber theorem. Here we prove that the pair $\left(C_{N}(G) \otimes_{t} C_{N}\left(G^{\prime}\right), \mu\right)$ is a DGA-algebra where $\mu$ is the standard product of $C_{N}(G) \otimes C_{N}\left(G^{\prime}\right)$. Furthermore, the injection of the twisted Eilenberg-Zilber contraction is a DGA-algebra morphism and the projection and the homotopy operator satisfy other weaker multiplicative properties.

Key Words: Contraction; Eilenberg-Zilber theorem; Homological perturbation lemma; Simplicial groups; Twisted cartesian product.

2000 Mathematics Subject Classification: $\quad$ 55R20; $18 \mathrm{D} 99$.

\section{INTRODUCTION}

The Eilenberg-Zilber theorem (Eilenberg and Zilber, 1953; Eilenberg and Mac Lane, 1954) gives a contraction from the chain complex of a cartesian product of simplicial sets to the corresponding tensor product of chain complexes. The twisted Eilenberg-Zilber theorem (Shih, 1962) generalizes this situation to the case of twisted cartesian products. It is well-known that any simplicial set $X$ has canonically associated a normalized chain complex, $C_{N}(X)$, which is a DGA-coalgebra. In the case that $X$ is a group, then $C_{N}(X)$ is a DGA-algebra too. Let $F \times{ }_{\tau} B$ be a twisted cartesian product. The twisted Eilenberg-Zilber theorem establishes a contraction from $C_{N}\left(F \times{ }_{\tau} B\right)$ to the twisted tensor product (in the sense of Brown, 1959) $C_{N}(F) \otimes_{t} C_{N}(B)$ :

$$
T E Z:\left\{C_{N}\left(F \times{ }_{\tau} B\right), C_{N}(F) \otimes_{t} C_{N}(B), f, g, \phi\right\}
$$

As a module, $C_{N}(F) \otimes_{t} C_{N}(B)$ is the ordinary tensor product of $C_{N}(F)$ with $C_{N}(B)$; both of which are DGA-algebras, when $F$ and $B$ are simplicial groups. Therefore,

Address correspondence to Prof. J. A. Armario, Departamento de Matemática Aplicada I, ETSII, Universidad de Sevilla, Avda. Reina Mercedes, S.N. 41012 Sevilla, Spain; Fax: +34-954557878; E-mail: armario@us.es 
we can define a module map

$$
\mu: C_{N}(F) \otimes_{t} C_{N}(B) \otimes C_{N}(F) \otimes_{t} C_{N}(B) \rightarrow C_{N}(F) \otimes_{t} C_{N}(B)
$$

by $\mu=\left(\mu_{C_{N}(F)} \otimes \mu_{C_{N}(B)}\right)(1 \otimes T \otimes 1)$ where $\mu_{C_{N}(F)}$ and $\mu_{C_{N}(B)}$ are the products in $C_{N}(F)$ and $C_{N}(B)$, respectively, and $T(x \otimes y)=(-1)^{|x||y|} y \otimes x$. Assuming that $F$ and $B$ are simplicial groups, the following question arises in a natural way: Is $C_{N}(F) \otimes_{t}$ $C_{N}(B)$ a DGA-algebra with regards to the product $\mu$ defined above?

In this article, under the hypothesis that $F \times_{\tau} B$ is a simplicial group, we give an affirmative answer to the question above. Furthermore, we prove that the injection of the contraction above, $g: C_{N}(F) \otimes_{t} C_{N}(B) \rightarrow C_{N}\left(F \times{ }_{\tau} B\right)$ is a DGAalgebra morphism. To this end, we take the work of Shih (1962) as starting point.

The analogous question for the coalgebra structure has been treated in Szcsarba (1961), but only the case for twisted cartesian products with base a sphere. In Kadeishvili and Saneblidze (2005), working with cubical sets, more progress in this direction has been shown. It is well-known that the study of the coalgebra structure has important consequences for describing $H^{*}\left(F \times{ }_{\tau} B\right)$ as an algebra (see Szcsarba, 1961). Nevertheless, until now, there is a lack of results in the literature about the algebra structure on $C_{N}(F) \otimes_{t} C_{N}(B)$. As we show at the end of this article, this structure is useful in some cases for achieving homological computations in an economical way. Here, we deal only with the algebra structure on $C_{N}(F) \otimes_{t} C_{N}(B)$.

Our approach for proving the main statement of this article deeply depends upon a suitable development of the homological perturbation theory (HPT) (Gugenheim and Lambe, 1989; Gugenheim et al., 1991), particularized to the category of DGA-algebras (Real, 2000). The cornerstone of HPT is the Basic Perturbation Lemma (BPL), which gives an explicit formula for transferring a perturbation of the differential of a chain complex to an equivalent chain subcomplex. In Huebschmann and Kadeishvili (1991) and Gugenheim et al. (1991) it is proved that the BPL also holds in the algebra category, assuming on the data the "maximum" degree of the compatibility with regards to the algebra structures. In Real (2000), assuming weaker hypotheses for the data involved in the BPL, an analogous result for the algebra category is obtained where the resulting contraction has weaker properties too. This result is stated in Theorem 3.2 and will be essential in this article.

The article is organized as follows. In Section 2 we introduce the basic notation and terminology used throughout the article, we define the objects studied in HPT, and sketch a familiar example which tells us that the Eilenberg-Zilber contraction can be perturbed into the twisted Eilenberg-Zilber contraction using BPL (see Shih, 1962). Section 3 is devoted to extend the notion of contraction to the category of DGA-algebras. In particular, we introduce the category of semi-full algebra contractions (we follow Real, 2000). Moreover, we show that the Eilenberg-Zilber contractions associated to two simplicial groups $F$ and $B$ is an almost-full algebra contraction. Furthermore, provided that $F \times_{\tau} B$ is a simplicial group, then the perturbation datum $\delta$ (which gives rise to the twisted Eilenberg-Zilber contraction by means of the BPL) is a derivation. Hence, as a consequence we obtain the main result of this article: $C_{N}(F) \otimes_{t} C_{N}(B)$ is a DGA-algebra with product $\mu$ and $g$ is morphism of DGA-algebra. In Section 4 we give an example where an algebra homological model for a particular twisted cartesian product is computed. 


\section{PRELIMINARIES}

We will quickly review basic notions of Homological Algebra specific to HPT, and introduce the notation and terminology that we use throughout the remainder of this article. More details can be found in Mac Lane (1995). Let $\Lambda$ be a commutative ring with nonzero unit, taken henceforth as ground ring and fixed throughout, and $A$ be an augmented differential graded algebra over $\Lambda$, briefly DGA-algebra. The differential, product, augmentation, and coaugmentation of $A$ will be denoted respectively by $d_{A}, \mu_{A}, \epsilon_{A}$, and $\eta_{A}$. Nevertheless, sometimes, we will write them simply $d, \mu, \epsilon$, and $\eta$ when no confusion can arise. In what follows, the Koszul sign conventions will be used. A morphism $\rho: A_{*} \rightarrow A_{*-1}$ is called derivation if it is compatible with the algebra structures on $A$. The degree of an element $a \in A$ is denoted by $|a|$. Also we recall that if $B$ is a DGA-algebra too, then $A \otimes B$ has canonically associated an algebra structure by means of the morphism $\mu_{A \otimes B}=\left(\mu_{A} \otimes \mu_{B}\right)\left(1_{A} \otimes T \otimes 1_{B}\right)$, where $T(b \otimes a)=(-1)^{|b||a|} a \otimes b$. If the DG-algebra $A$ is connected, that is $A_{0}=\Lambda$ and $d_{1}: A_{1} \rightarrow A_{0}$ is zero, then there is a canonical augmentation $\epsilon_{A}=1_{\Lambda}: A_{0} \rightarrow \Lambda$.

We will use here the twisted tensor product structure. Let $A$ be a DG-algebra and $C$ be a DG-coalgebra $\left(\Delta_{C}\right.$ denotes its coproduct). A twisting cochain is a morphism of graded modules $t: C_{*} \rightarrow A_{*^{-} 1}$ such that

$$
d_{A} t+t d_{C}+t \cup t=0, \quad \epsilon_{A} t=0, \quad t \eta_{C}=0 ;
$$

where $t \cup t=-\mu_{A}(t \otimes t) \Delta_{C}$. Given the DGA-A-module $M$, the DGA-C-comodule $N$, and a twisting cochain $t$ it is well-known that $d^{t}=d_{M} \otimes 1+1 \otimes d_{N}+t \cap$ is a differential on $M \otimes N$, where the morphism $t \cap$ is defined by

$$
t \cap=\left(\mu_{M} \otimes 1\right)(1 \otimes t \otimes 1)\left(1 \otimes \Delta_{N}\right)
$$

The DG-module $\left(M \otimes N, d^{t}\right)$ is called the twisted tensor product (or TTP) of $M$ and $N$ along $t$. We will also use the notation $M \otimes_{t} N$ for such a DG-module.

Now, we define precisely the objects studied in homological perturbation theory and sketch a familiar example.

A contraction (see Eilenberg and Mac Lane, 1953; Huebschmann and Kadeishvili, 1991) is a data set $c:\{N, M, f, g, \phi\}$ where $f: N \rightarrow M$ and $g: M \rightarrow N$ are morphisms of DG-modules (called, respectively, the projection and the inclusion) and $\phi: N \rightarrow N$ is a morphism of graded modules of degree +1 (called the homotopy operator). These data are required to satisfy the rules: (c1) $f g=1_{M}$, (c2) $\phi d_{N}+$ $d_{N} \phi+g f=1_{N}$, (c3) $\phi \phi=0$, (c4) $\phi g=0$, and (c5) $f \phi=0$. These last three are called the side conditions (Lambe and Stasheff, 1987). In fact, these may always be assumed to hold, since the homotopy $\phi$ can be altered to satisfy these conditions (Gugenheim and Lambe, 1989). These formulas have a simple interpretation: the DG-module $M$, the small one, is isomorphic to a subDG-module of $N$, the big one, and a decomposition $N=M \oplus L$ is given where the summand $L$ is acyclic. This implies that both chain complexes $N$ and $M$ have the same homology. We will also denote a contraction $c$ by $(f, g, \phi): N \Rightarrow M$.

If we have two contractions $\left(f_{i}, g_{i}, \phi_{i}\right)$ from $N_{i}$ to $M_{i}$, for $i=1,2$, then the following contractions can be constructed (see Eilenberg and Mac Lane, 
1953):

- The tensor product contraction $\left(f_{2} \otimes f_{1}, g_{1} \otimes g_{2}, \phi_{1} \otimes g_{2} f_{2}+1_{N_{1}} \otimes \phi_{2}\right)$ from $N_{1} \otimes N_{2}$ to $M_{1} \otimes M_{2}$

- If $N_{2}=M_{1}$, the composition contraction $\left(f_{2} f_{1}, g_{1} g_{2}, \phi_{1}+g_{1} \phi_{2} f_{1}\right)$ from $N_{1}$ to $M_{2}$;

The Eilenberg-Zilber theorem (Eilenberg and Zilber, 1953) provides the most classic example of a contraction of chain complexes. An Eilenberg-Zilber contraction is defined in Eilenberg and Mac Lane (1954) by the data set

$$
E Z_{F, B}:\left\{C_{N}(F \times B), C_{N}(F) \otimes C_{N}(B), A W_{F, B}, E M L_{F, B}, S H I_{F, B}\right\},
$$

where $F$ and $B$ are simplicial sets. The results of the present article depend heavily on these morphisms. We follow May's (1967) book for the terminology and notation on Simplicial Topology.

One of the cornerstones of homological perturbation theory is the BPL. It provides a beautiful way of unifying many disparate results in algebraic topology concerning chain homotopy equivalences, and it can be used to find new results as well.

Now, we recall the concept of a perturbation datum. Let $N$ be a graded module and let $f: N \rightarrow N$ be a morphism of graded modules. The morphism $f$ is pointwise nilpotent if for all $x \in N(x \neq 0)$, a positive integer $n$ exists (in general, the number $n$ depends on the element $x$ ) such that $f^{n}(x)=0$. A perturbation of $a$ $D G$-module $N$ is a morphism of graded modules $\delta: N \rightarrow N$ of degree -1 , such that $\left(d_{N}+\delta\right)^{2}=0$ and $\delta_{1}=0$. A perturbation datum of the contraction $c:\{N, M, f, g, \phi\}$ is a perturbation $\delta$ of the DGA-module $N$ verifying that the composition $\phi \delta$ is pointwise nilpotent.

A Transference Problem consists of a contraction $c:\{M, N, f, g, \phi\}$ together with a perturbation $\delta$ of the DG-module $N$. The problem is to determine new morphisms $d_{\delta}, f_{\delta}, g_{\delta}$, and $\phi_{\delta}$ such that $c_{\delta}:\left\{\left(N, d_{N}+\delta\right),\left(M, d_{M}+d_{\delta}\right), f_{\delta}, g_{\delta}, \phi_{\delta}\right\}$ is a contraction.

The BPL (Brown, 1967; Gugenheim and Lambe, 1989; Gugenheim et al., 1991; Real, 2000) gives an explicit solution to the Transference Problem, assuming that $\delta$ is a perturbation datum of $c$.

Theorem 2.1 (BPL). Let $c:\{N, M, f, g, \phi\}$ be a contraction and $\delta: N \rightarrow N a$ perturbation datum of $c$. Then, a new contraction

$$
c_{\delta}:\left\{\left(N, d_{N}+\delta\right),\left(M, d_{M}+d_{\delta}\right), f_{\delta}, g_{\delta}, \phi_{\delta}\right\}
$$

is defined by the formulas: $d_{\delta}=f \delta \Sigma_{c}^{\delta} g, f_{\delta}=f\left(1-\delta \Sigma_{c}^{\delta} \phi\right), g_{\delta}=\Sigma_{c}^{\delta} g, \phi_{\delta}=\Sigma_{c}^{\delta} \phi$, where

$$
\Sigma_{c}^{\delta}=\sum_{i \geq 0}(-1)^{i}(\phi \delta)^{i}=1-\phi \delta+\phi \delta \phi \delta-\cdots+(-1)^{i}(\phi \delta)^{i}+\cdots .
$$

Let us note that $\Sigma_{c}^{\delta}(x)$ is a finite sum for each $x \in N$, because of the pointwise nilpotency of the composition $\phi \delta$. Moreover, it is obvious that the morphism $d_{\delta}$ is a perturbation of the DG-module $\left(M, d_{M}\right)$.

The Twisted Eilenberg-Zilber theorem can be seen as an important example of the usefulness of this lemma (see Shih, 1962). It solves the Transference Problem 
for twisted cartesian products. In the sequel we give the ingredients for a concrete statement of this result.

Consider two simplicial sets $F, B$ and a simplicial group $G$ which operates on $F$ from the left. A twisted cartesian product $E$ with fibre $F$, base $B$, and structural group $G$ consists of a simplicial set $E_{n}=F_{n} \times B_{n}$ and

$$
\begin{aligned}
\partial_{0}(f, b) & =\left(\tau b * \partial_{0} f, \partial_{0} b\right) \\
\partial_{i}(f, b) & =\left(\partial_{i} f, \partial_{i} b\right), \quad \text { for } i>0 \\
s_{i}(f, b) & =\left(s_{i} f, s_{i} b\right), \quad \text { for } i \geq 0
\end{aligned}
$$

as face and degeneracy operators. Here $*: G \times F \rightarrow F$ is the action of $G$ on $F$ and $\tau$ is a twisting function, i.e., $\tau_{n}: B_{n} \rightarrow G_{n-1}, n \geq 1$ satisfies

$$
\begin{aligned}
& \partial_{0} \tau(b)=\left[\tau\left(\partial_{0} b\right)\right]^{-1} \cdot \tau\left(\partial_{1} b\right) \\
& \partial_{i} \tau(b)=\tau\left(\partial_{i+1} b\right), \quad \text { for } i>0 \\
& s_{i} \tau(b)=\tau\left(s_{i+1} b\right), \quad \text { for } i \geq 0 \\
& \tau\left(s_{0} b\right)=e_{n},
\end{aligned}
$$

where $e_{n}$ denotes the identity element of the corresponding group $G_{n}$. We write $E=F \times{ }_{\tau} B$.

Theorem 2.2 (Shih, 1962, Twisted Eilenberg-Zilber Theorem). The morphism

$$
\delta(f, b)=\left(\tau b * \partial_{0} f, \partial_{0} b\right)-\left(\partial_{0} f, \partial_{0} b\right), \quad(f, b) \in C_{N}(F \times B)
$$

is a perturbation datum of the contraction,

$$
E Z_{F, B}:\left\{C_{N}(F \times B), C_{N}(F) \otimes C_{N}(B), A W_{F, B}, E M L_{F, B}, S H I_{F, B}\right\} .
$$

From these data a new contraction (called the twisted Eilenberg-Zilber contraction) is obtained by applying BPL:

$$
T E Z_{F, B}:\left\{C_{N}\left(F \times_{\tau} B\right),\left(C_{N}(F) \otimes C_{N}(B), d^{\otimes}+d_{\delta}\right),\left(A W_{F, B}\right)_{\delta},\left(E M L_{F, B}\right)_{\delta},\left(S H I_{F, B}\right)_{\delta}\right\},
$$

where the smaller chain complex consists of a twisted tensor product along the twisting cochain $t, C_{N}(F) \otimes_{t} C_{N}(B)$, for $t=p \circ d_{\delta} \circ \rho$

$$
C_{N}(B) \stackrel{\rho}{\longrightarrow} C_{N}(G) \otimes C_{N}(B) \stackrel{d_{\delta}}{\longrightarrow} C_{N}(G) \otimes C_{N}(B) \stackrel{p}{\longrightarrow} C_{N}(G)
$$

where

$$
\rho(x)=e_{0} \otimes x, \quad e_{0} \text { being the identity element of } G_{0} \text { and } p(y \otimes x)=y \cdot \epsilon_{C_{N}(B)} x .
$$

Hence,

$$
d_{\delta}=t \cap=\left(\mu_{C_{N}(F)} \otimes 1\right)(1 \otimes t \otimes 1)\left(1 \otimes \Delta_{C_{N}(B)}\right),
$$

where $\mu_{C_{N}(F)}$ is the module action induced by the the action $*: G \times F \rightarrow F$. 


\section{THE MAIN THEOREMS}

When solving problems in homological or homotopical algebra, one often works with chain complexes with additional algebraic structure, e.g., chain algebras or coalgebras. For instance, given a simplicial group $G$, one may define a product $\mu_{C_{N}(G)}$ on $C_{N}(G)$, called the Eilenberg-Mac Lane or Pontrjagin product, by composing the Eilenberg-Mac Lane operator $E M L_{G, G}$ and the morphism of DG-modules induced by the product on $G, \cdot: C_{N}(G \times G) \rightarrow C_{N}(G)$. An explicit formula for this morphism is

$$
\mu_{C_{N}(G)}\left(x_{p} \otimes y_{q}\right)=\sum_{(\alpha, \beta)}(-1)^{s g(\alpha, \beta)}\left(s_{\beta_{q}} \ldots s_{\beta_{1}} x_{p} \cdot s_{\alpha_{p}} \ldots s_{\alpha_{1}} y_{q}\right)
$$

where the $(\alpha, \beta)$ index runs over all the $(p, q)$-shuffles. The pair $\left(C_{N}(G), \mu_{C_{N}(G)}\right)$ is a DGA-algebra, which is commutative whenever $G$ is. Furthermore, $\left(C_{N}(G), \mu_{C_{N}(G)}, \Delta_{C_{N}(G)}\right)$ is a Hopf DGA-algebra where $\Delta_{C_{N}(G)}$ is the AlexanderWhitney coproduct (see May, 1967, p. 138). It is natural to extend the notion of contraction to the proper categories of such objects. Here, we are only interested in the category of DGA-algebras.

Let $A$ and $A^{\prime}$ be two DGA-algebras. Given a contraction $c:\left\{A, A^{\prime}, f, g, \phi\right\}$ we can distinguish different types of algebra contractions in function of the degree of compatibility of the morphisms $f, g$ and $\phi$ with regards to the products $\mu_{A}$ and $\mu_{A^{\prime}}$. We say (see Real, 2000) that $c$ is:

(i) A full algebra contraction if $f$ and $g$ are morphisms of DGA-algebras and $\phi$ is an algebra homotopy;

(ii) An almost-full algebra contraction if $f$ and $g$ are morphisms of DGA-algebras and $\phi$ is a quasi algebra homotopy;

(iii) A semi-full algebra contraction if $f$ is a quasi algebra projection, $g$ is a morphism of DGA-algebras and $\phi$ is a quasi algebra homotopy.

Here the projection $f$ is said to be a quasi algebra projection if the following conditions hold:

$$
f \mu_{A}(\phi \otimes \phi)=0, \quad f \mu_{A}(\phi \otimes g)=0, \quad f \mu_{A}(g \otimes \phi)=0 .
$$

The homotopy operator $\phi$ is said to be a quasi algebra homotopy if the following conditions hold:

$$
\phi \mu_{A}(\phi \otimes \phi)=0, \quad \phi \mu_{A}(\phi \otimes g)=0, \quad \phi \mu_{A}(\phi \otimes \phi)=0 .
$$

We also recall that $\phi$ is called a homotopy algebra if

$$
\phi \mu_{A}=\mu_{A} \phi^{[\otimes 2]}
$$

where $\phi^{[\otimes 2]}=1 \otimes \phi+\phi \otimes g f$. 
One can immediately see that, in the context of algebra contractions, the designation of full implies almost-full, as well as almost-full implies semi-full. Let us observe that each of these classes is closed under composition and tensor product of contractions. Examples of all these types of contractions appear in Real (2000). Furthermore, some interesting properties concerning the relationship between these categories are given in Real (2000). For instance,

Proposition 3.1 (Real, 2000). An almost-full algebra contraction $c:\left\{A, A^{\prime}, f, g, \phi\right\}$ is a full algebra contraction if and only if $\phi \mu_{A}(1 \otimes \phi)=0$.

The following result tells us that the set of semi-full algebra contractions is closed under homological perturbation. This theorem is used in the proof of one of the main theorems of this article.

Theorem 3.2 (Real, 2000, SF-APL). Taking as input data a semi-full algebra contraction $c:\left\{A, A^{\prime}, f, g, \phi\right\}$ and an algebra perturbation datum $\delta$ of $c$, the perturbed contraction $c_{\delta}$ is a semi-full algebra contraction, where the product on $A_{\delta}^{\prime}$ is the original product $\mu_{A^{\prime}}$.

Before stating one of the main results of this article, we recall that the cartesian product of two simplicial groups $G \times G^{\prime}$ is again a simplicial group with the trivial (internal) law for the group, so that the formula for the product $\mu_{C_{N}\left(G \times G^{\prime}\right)}$ on $C_{N}\left(G \times G^{\prime}\right)$ is

$$
\mu_{C_{N}\left(G \times G^{\prime}\right)}=(\cdot \times \cdot)(1 \times t \times 1) E M L_{G \times G^{\prime}, G \times G^{\prime}} .
$$

Here operator $t$ is defined so that it interchanges the components of a cartesian product of simplicial sets: $t(x \times y)=y \times x$.

The next theorem yields an important example of an almost-full algebra contraction which is not full in general. The proof that it is not a full algebra contraction is by means of a construction of a particular cartesian product where we show that $\phi \mu_{A}(1 \otimes \phi)$ is not the null morphism. This example is included after the proof of the following result.

Theorem 3.3. Let $G$ and $G^{\prime}$ be simplicial groups. The Eilenberg-Zilber contraction associated to $G$ and $G^{\prime}$

$$
E Z_{G, G^{\prime}}:\left\{C_{N}\left(G \times G^{\prime}\right), C_{N}(G) \otimes C_{N}\left(G^{\prime}\right), A W_{G, G^{\prime}}, E M L_{G, G^{\prime}}, S H I_{G, G^{\prime}}\right\}
$$

is an almost-full algebra contraction.

Proof. For the sake of clarity we denote the projection, inclusion and homotopy operators of the contraction (2) by $f, g$ and $\phi$, respectively.

Under the hypothesis that $G$ and $G^{\prime}$ are commutative, Eilenberg and Mac Lane (1954, Theorem 3.2) proved that the operators $f$ and $g$ are both morphisms of DGA-algebras; that is, the following identities hold:

$$
f \mu_{C_{N}\left(G \times G^{\prime}\right)}=\mu_{C_{N}(G) \otimes C_{N}\left(G^{\prime}\right)}(f \otimes f), \quad g \mu_{C_{N}(G) \otimes C_{N}\left(G^{\prime}\right)}=\mu_{C_{N}\left(G \times G^{\prime}\right)}(g \otimes g) .
$$


This result may be extended to the noncommutative framework, as we show as follows.

For $f$, this is immediate since a careful reading of the proof of EilenbergMac Lane shows that the commutative assumption is not used in this case. However, Eilenberg-Mac Lane's proof of the multiplicative behaviour of $g$ uses the commutative condition, so we have to take an alternative way. The following results will lead us to the desired goal.

It is straightforward that if $(\alpha, \beta)$ is a $(p, q)$-shuffle, then $(\beta, \alpha)$ is a $(q, p)$ shuffle. Furthermore, their respective signatures verify the following identity:

$$
\operatorname{sg}(\alpha, \beta)+\operatorname{sg}(\beta, \alpha)=p q
$$

In the sequel $a$ denotes an element of $G_{p}, a^{\prime}$ denotes an element of $G_{r}^{\prime}$ and 1, 1' denote the identity elements of $G_{*}$ and $G_{*}^{\prime}$, respectively, in the appropriate degree such that all the cartesian products which appear below are well defined. As a consequence of (3), we have

$$
\mu_{C_{N}\left(G \times G^{\prime}\right)}\left(\left(a \times 1^{\prime}\right) \otimes\left(1 \times a^{\prime}\right)\right)=(-1)^{p r} \mu_{C_{N}\left(G \times G^{\prime}\right)}\left(\left(1 \times a^{\prime}\right) \otimes\left(a \times 1^{\prime}\right)\right) .
$$

Moreover, it may be readily checked that the following identity holds:

$$
\mu_{C_{N}\left(G \times G^{\prime}\right)}\left(\left(a \times 1^{\prime}\right) \otimes\left(1 \times a^{\prime}\right)\right)=g\left(a \otimes a^{\prime}\right) .
$$

As it is shown in Eilenberg and Mac Lane (1954, p. 56), both the DGA-algebras $C_{N}(G)$ and $C_{N}\left(G^{\prime}\right)$ may be considered as subalgebras of $C_{N}\left(G \times G^{\prime}\right)$. In this way, we sometimes identify some elements $a$ and $a^{\prime}$ with their correspondents $a \times 1^{\prime}$ and $1 \times a^{\prime}$, respectively. Furthermore, a rule connecting those products of the algebras above is

$$
\mu_{C_{N}(G)}(a \otimes b)=\mu_{C_{N}\left(G \times G^{\prime}\right)}\left(\left(a \times 1^{\prime}\right) \otimes\left(b \times 1^{\prime}\right)\right)
$$

for any $a \in G_{p}$ and $b \in G_{q}$. Analogously,

$$
\mu_{C_{N}(G)}\left(a^{\prime} \otimes b^{\prime}\right)=\mu_{C_{N}\left(G \times G^{\prime}\right)}\left(\left(1 \times a^{\prime}\right) \otimes\left(1 \times b^{\prime}\right)\right)
$$

for any $a^{\prime} \in G_{p}^{\prime}$ and $b^{\prime} \in G_{q}^{\prime}$.

Now it is possible to prove the multiplicative character of the inclusion morphism $g$, that is,

$$
g\left(\mu_{C_{N}(G) \otimes C_{N}\left(G^{\prime}\right)}\left(\left(a \otimes a^{\prime}\right) \otimes\left(b \otimes b^{\prime}\right)\right)\right)=\mu_{C_{N}\left(G \times G^{\prime}\right)}\left(g\left(a \otimes a^{\prime}\right) \otimes g\left(b \otimes b^{\prime}\right)\right),
$$

where $a \in G_{p}, b \in G_{q}, a^{\prime} \in G_{r}^{\prime}$, and $b^{\prime} \in G_{s}^{\prime}$. Working with the term on the left,

$$
g\left(\mu_{C_{N}(G) \otimes C_{N}\left(G^{\prime}\right)}\left(\left(a \otimes a^{\prime}\right) \otimes\left(b \otimes b^{\prime}\right)\right)\right)=g\left((-1)^{q r} \mu_{C_{N}(G)}(a \otimes b) \otimes \mu_{C_{N}\left(G^{\prime}\right)}\left(a^{\prime} \otimes b^{\prime}\right)\right)
$$

(attending to (5), (6), and (7))

$$
=(-1)^{q r} \mu_{C_{N}\left(G \times G^{\prime}\right)}\left(\mu_{C_{N}\left(G \times G^{\prime}\right)}\left(a \times 1^{\prime} \otimes b \times 1^{\prime}\right) \otimes \mu_{C_{N}\left(G \times G^{\prime}\right)}\left(1 \times a^{\prime} \otimes 1 \times b^{\prime}\right)\right)
$$


(using the associativity of the product $\mu_{C_{N}\left(G \times G^{\prime}\right)}$ and (4))

$$
=\mu_{C_{N}\left(G \times G^{\prime}\right)}\left(\mu_{C_{N}\left(G \times G^{\prime}\right)}\left(a \times 1^{\prime} \otimes 1 \times a^{\prime}\right) \otimes \mu_{C_{N}\left(G \times G^{\prime}\right)}\left(b \times 1^{\prime} \otimes 1 \times b^{\prime}\right)\right)
$$

(by $(5))$

$$
=\mu_{C_{N}\left(G \times G^{\prime}\right)}\left(g\left(a \otimes a^{\prime}\right) \otimes g\left(b \otimes b^{\prime}\right)\right) .
$$

Then, we have just proved that both the projection and the injection operators of the inicial Eilenberg-Zilber contraction are morphisms of DGA-algebras. It remains to prove that the homotopy operator $\phi$ is a quasi-algebra homotopy in order to conclude that the Eilenberg-Zilber contraction from $C_{N}\left(G \times G^{\prime}\right)$ to $C_{N}(G) \otimes C_{N}\left(G^{\prime}\right)$ is an almost-full algebra contraction; that is,

$$
\begin{aligned}
\phi \mu_{C_{N}\left(G \times G^{\prime}\right)}(\phi \otimes \phi) & =0, \\
\phi \mu_{C_{N}\left(G \times G^{\prime}\right)}(\phi \otimes g) & =0, \\
\phi \mu_{C_{N}\left(G \times G^{\prime}\right)}(g \otimes \phi) & =0 .
\end{aligned}
$$

For the remainder of the proof $A$ and $A^{\prime}$ will denote $C_{*}^{N}\left(G \times G^{\prime}\right)$ and $C_{*}^{N}(G) \otimes$ $C_{*}^{N}\left(G^{\prime}\right)$, respectively. Obviously, $\mu_{A}$ and $\mu_{A^{\prime}}$ denote their respective products.

We set up some definitions, notations, and remarks that will be used in the sequel.

1. We deal with normalized chain complexes, so that zero means zero modulo degeneracies. In other words, any linear combination of degenerate elements must be considered zero.

2. A simplicial operator (or simplicial operator of groups) $D$ of type $(p, q), p$, and $q$ nonnegative integers, is a collection of maps (or homomorphisms) $D(X)$ : $X_{p} \rightarrow X_{q}$, one for each simplicial set (or simplicial group) $X$, natural with respect to simplicial maps (or simplicial homomorphisms). The integer $p-q$ is called the degree of D. In Szcsarba $(1961, \S 1)$ it is proved that any simplicial operator $D$ of type $(p, q)$ can be written uniquely as

$$
D=s_{i_{r}} \ldots s_{i_{1}} \partial_{j_{s}} \ldots \partial_{j_{1}}
$$

where $r-s=q-p, i_{r}>i_{r-1}>\cdots>i_{1}$, and $j_{s}<j_{s-1}<\cdots \leq p$. In the case that $D$ is a simplicial operator of groups, Eilenberg and Mac Lance stated in Eilenberg and Mac Lane $(1953, \S 3)$ that $D$ can be represented uniquely as a linear combination of terms like (8) with integral coefficients.

Given a simplicial operator $D$ of degree $r-s$ in the form of (8) and initial dimension $p$, the derived operator of $D$, denoted by $D^{\prime}$, is the simplicial operator of degree $r-s$ and initial dimension $p+1$ given by raising all the indices by 1 , i.e.,

$$
D^{\prime}=s_{i_{r}+1} \ldots s_{i_{1}+1} \partial_{j_{s}+1} \ldots \partial_{j_{1}+1} \text {. }
$$

This definition is straightforwardly extended by linearity to simplicial operator of groups. 
Now, we give some of the main properties concerning to the "derivation" extracted from Eilenberg and Mac Lane (1953, §3):

$$
\partial_{0} D^{\prime}=D \partial_{0} ; \quad s_{0} D=D^{\prime} s_{0}, \quad \text { if } \partial_{0} \text { is not in } D .
$$

If $D_{1}$ and $D_{2}$ are simplicial operators of groups, then

$$
\left(D_{1}+D_{2}\right)^{\prime}=D_{1}^{\prime}+D_{2}^{\prime}, \quad\left(D_{1} D_{2}\right)^{\prime}=D_{1}^{\prime} D_{2}^{\prime}, \quad 0^{\prime}=0, \quad 1^{\prime}=1,
$$

whenever the sum $D_{1}+D_{2}$ or the composite $D_{1} D_{2}$ is defined.

3. We denote $h=g f$ the morphism given by the composition of the injection and projection morphisms of the Eilenberg-Zilber contraction.

4. Recall that $f, g$ and $\phi$ verify the following basic rules, from the definition of contraction:

$$
f \phi=0, \quad \phi g=0, \quad \phi \phi=0 ;
$$

where the identities above are considered on normalized chain complexes.

5. Eilenberg and Mac Lane $(1953,1954)$ extended the notion of derivation to $g, h$, and $\phi$ in a natural way.

6. The homotopy operator $\phi$ is recursively defined by the formula:

$$
\begin{cases}\phi_{0}=0, & \text { on zero degree } \\ \phi_{n}=-\phi_{n-1}^{\prime}+h_{n}^{\prime} s_{0}, & \text { on } n>0 \text { degree }\end{cases}
$$

as it is shown in Eilenberg and Mac Lane (1954). rule:

7. Eilenberg-Mac Lane also proved that the injection operator verifies the

$$
g=g^{\prime}\left(s_{0} \otimes 1+1 \otimes s_{0}\right) .
$$

Furthermore, it is easy to check that the following identities hold:

$$
\begin{aligned}
s_{0} g & =g^{\prime}\left(s_{0} \otimes s_{0}\right), \\
s_{0} \phi & =\phi^{\prime} s_{0} .
\end{aligned}
$$

8. It follows at once from (10) and (11) that:

$$
f \phi^{\prime}=f h^{\prime} s_{0},
$$

since, $f \phi=0 \Rightarrow 0=f\left(-\phi^{\prime}+h^{\prime} s_{0}\right)=-f \phi^{\prime}+f h^{\prime} s_{0}$.

Attending to the remarks above, we are now able to prove that the homotopy operator $\phi$ constitutes a quasi-algebra homotopy. Firstly, we prove that

$$
\phi \mu_{A}(\phi \otimes g)=0 .
$$


The proof is by induction on the degree of the homotopy operator $\phi$ placed on the left.

On degree one the identity (16) is verified by definition, attending to (11):

$$
\phi_{1} \mu_{A}\left(\phi_{0} \otimes g_{0}\right)=0
$$

Let us suppose that (16) is satisfied on degree $k$, for all $k$ less than $n$. Our aim is to verify the corresponding identity on degree $n$. In order to apply the induction hypothesis, we use (11) to substitute $\phi_{n}$ by $-\phi_{n-1}^{\prime}+h_{n}^{\prime} s_{0}$.

Recall that the product $\mu_{A}=\mu_{C_{N}\left(G \times G^{\prime}\right)}$ is defined by,

$$
\mu_{A}=(\cdot \times \cdot)(1 \times t \times 1) g_{G \times G^{\prime}, G \times G^{\prime}}
$$

in terms of the Eilenberg-Zilber operator. The notation $\bar{g}=g_{G \times G^{\prime}, G \times G^{\prime}}$ simplifies the reading and writing for the remainder of the article.

Hence, the formula (16) on degree $n$ can be rewritten as:

$$
\begin{aligned}
\phi_{n} \mu_{A}(\phi \otimes g)= & \left(-\phi_{n-1}^{\prime}+h_{n}^{\prime} s_{0}\right) \mu_{A}\left[\left(-\phi^{\prime}+h^{\prime} s_{0}\right) \otimes g\right] \\
= & \left(-\phi_{n-1}^{\prime}+h_{n}^{\prime} s_{0}\right)(\cdot \times \cdot)(1 \times t \times 1) \bar{g}_{n-1}^{\prime} \\
& \times\left(s_{0} \otimes 1+1 \otimes s_{0}\right)\left[\left(-\phi^{\prime}+h^{\prime} s_{0}\right) \otimes g\right] \\
= & \phi_{n-1}^{\prime}(\cdot \times \cdot)(1 \times t \times 1) \bar{g}_{n-1}^{\prime}\left(s_{0} \phi^{\prime} \otimes g\right) \\
& -\phi_{n-1}^{\prime}(\cdot \times \cdot)(1 \times t \times 1) \bar{g}_{n-1}^{\prime}\left(s_{0} h^{\prime} s_{0} \otimes g\right) \\
& +\phi_{n-1}^{\prime}(\cdot \times \cdot)(1 \times t \times 1) \bar{g}_{n-1}^{\prime}\left(\phi^{\prime} \otimes s_{0} g\right) \\
& -\phi_{n-1}^{\prime}(\cdot \times \cdot)(1 \times t \times 1) \bar{g}_{n-1}^{\prime}\left(h^{\prime} s_{0} \otimes s_{0} g\right) \\
& +h_{n}^{\prime} s_{0}(\cdot \times \cdot)(1 \times t \times 1) \bar{g}_{n}(\phi \otimes g) .
\end{aligned}
$$

In the sequel we shall prove that each of these summands vanish modulo degeneracies.

- On one hand, since $\partial_{0} \notin \phi^{\prime}$, it follows at once that $s_{0} \phi^{\prime}=\phi^{\prime \prime} s_{0}$; taking derived operators in (11),

$$
\phi^{\prime}=-\phi^{\prime \prime}+h^{\prime \prime} s_{1} \Rightarrow \phi^{\prime \prime}=-\phi^{\prime}+h^{\prime \prime} s_{1} \Rightarrow \phi^{\prime \prime} s_{0}+\phi^{\prime} s_{0}=h^{\prime} s_{1} s_{0} .
$$

On the other hand, since $\partial_{0} \notin h^{\prime}$, it is verified that $s_{0} h^{\prime} s_{0}=h^{\prime \prime} s_{1} s_{0}$. Thus, $\phi^{\prime \prime} s_{0}+$ $\phi^{\prime} s_{0}=s_{0} h^{\prime} s_{0}$.

Hence, on substituting the value of $s_{0} h^{\prime} s_{0}$ into (17) and carrying out the indicated additions, it can be rewritten as

$$
-\phi_{n-1}^{\prime}(\cdot \times \cdot)(1 \times t \times 1) \bar{g}_{n-1}^{\prime}\left(\phi^{\prime} s_{0} \otimes g\right)
$$

(using (12)),

$$
=-\phi_{n-1}^{\prime}(\cdot \times \cdot)(1 \times t \times 1) \bar{g}_{n-1}^{\prime}\left[\phi^{\prime} s_{0} \otimes g^{\prime}\left(s_{0} \otimes 1+1 \otimes s_{0}\right)\right],
$$


taking into account that $\phi_{n-1}^{\prime}(\cdot \times \cdot)(1 \times t \times 1) \bar{g}_{n-1}^{\prime}\left(\phi^{\prime} \otimes g^{\prime}\right)=\left(\phi_{n-1} \mu_{A}(\phi \otimes g)\right)^{\prime}$ by the definition of $\mu_{A}$ and the second property of (9). Now using the induction hypothesis and the third property of (9),

$$
\left(\phi_{n-1} \mu_{A}(\phi \otimes g)\right)^{\prime}=0 .
$$

Hence, the summand (17) vanishes as desired.

- The fact that (18) is also zero follows at once from the identity $s_{0} g=g^{\prime}\left(s_{0} \otimes s_{0}\right)$ and the induction hypothesis.

- Taking into account that $g$ is a DGA-algebra morphism and the property $\phi g=0$, we conclude that

$$
\phi \mu_{A}(g \otimes g)=0,
$$

and as a straightforward consequence $\phi^{\prime} \mu_{A}^{\prime}\left(g^{\prime} \otimes g^{\prime}\right)=0$. Furthermore, it is verified that

$$
h^{\prime}=g^{\prime} f^{\prime} \quad \text { and } \quad s_{0} g=g^{\prime}\left(s_{0} \otimes s_{0}\right) .
$$

Using these identities and following similar arguments to those for the summands before, we conclude that the summand (19) vanishes as well.

- Concerning the summand (20) the process is slightly more complicated. It is easy to check that

$$
\begin{aligned}
h^{\prime} s_{0}(\cdot \times \cdot)(1 \times t \times 1) \bar{g}(\phi \otimes g) & =h^{\prime}(\cdot \times \cdot)(1 \times t \times 1) s_{0} \bar{g}(\phi \otimes g) \\
& =h^{\prime}(\cdot \times \cdot)(1 \times t \times 1) \bar{g}^{\prime}\left(s_{0} \otimes s_{0}\right)(\phi \otimes g) \\
& =h^{\prime}(\cdot \times \cdot)(1 \times t \times 1) \bar{g}^{\prime}\left(s_{0} \phi \otimes s_{0} g\right) .
\end{aligned}
$$

The identity

$$
h \mu_{A}=g f \mu_{A}=g \mu_{A^{\prime}}(f \otimes f)
$$

follows at once from the fact that the projection operator $f$ is a morphism of DGA-algebras. Now, considering derived morphisms

$$
h^{\prime}\left(\mu_{A}\right)^{\prime}=g^{\prime} f^{\prime}\left(\mu_{A}\right)^{\prime}=g^{\prime}\left(\mu_{A^{\prime}}\right)^{\prime}\left(f^{\prime} \otimes f^{\prime}\right),
$$

that is,

$$
h^{\prime}(\cdot \times \cdot)(1 \times t \times 1) \bar{g}^{\prime}=g^{\prime}\left(\mu_{A^{\prime}}\right)^{\prime}\left(f^{\prime} \otimes f^{\prime}\right) .
$$

By (11),

$$
s_{0} \phi=s_{0}\left(-\phi^{\prime}+h^{\prime} s_{0}\right)=-\phi^{\prime \prime} s_{0}+h^{\prime \prime} s_{0} s_{0}=-\phi^{\prime \prime} s_{0}+h^{\prime \prime} s_{1} s_{0},
$$

since $\partial_{0} \notin \phi^{\prime}, \partial_{0} \notin h^{\prime}$, and $s_{0} s_{0}=s_{1} s_{0}$. 
On the other hand,

$$
\begin{aligned}
f^{\prime}\left(-\phi^{\prime \prime} s_{0}+h^{\prime \prime} s_{1} s_{0}\right) & =-f^{\prime} \phi^{\prime \prime} s_{0}+f^{\prime} h^{\prime \prime} s_{1} s_{0} \\
& =\left(-f^{\prime} \phi^{\prime \prime}+f^{\prime} h^{\prime \prime} s_{1}\right) s_{0} \\
& =\left(-f \phi^{\prime}+f h^{\prime} s_{0}\right)^{\prime} s_{0} .
\end{aligned}
$$

Combining (21), (23), (22), (24), and (13) the summand (20) can be rewritten as

$$
g^{\prime}\left(\mu_{A^{\prime}}\right)^{\prime}\left[\left(-f \phi^{\prime}+f h^{\prime} s_{0}\right)^{\prime} \otimes f^{\prime} g^{\prime}\right]\left[s_{0} \otimes\left(s_{0} \otimes s_{0}\right)\right] .
$$

Next we prove

$$
g \mu_{A^{\prime}}\left[\left(-f \phi^{\prime}+f h^{\prime} s_{0}\right) \otimes f g\right]=0
$$

and as a direct consequence simply taking derived operators we have that (25) vanishes too.

By (15),

$$
-f \phi^{\prime}+f h^{\prime} s_{0}=0, \quad \text { (modulo degeneracies) }
$$

and the product $\mu_{A^{\prime}}$ maps degeneracies into degeneracies, since

$$
\mu_{A^{\prime}}=\left(\mu_{C_{N}(G)} \otimes \mu_{C_{N}\left(G^{\prime}\right)}\right)(1 \otimes T \otimes 1)=(\cdot \otimes \cdot)\left(g_{G} \otimes g_{G^{\prime}}\right)(1 \otimes T \otimes 1),
$$

and the injection operator $g$ maps degeneracies into degeneracies (see Eilenberg and Mac Lane, 1953, Lemma 5.3). Thus, (25) is satisfied. This completes the proof of (16).

The identities,

$$
\phi \mu_{A}(g \otimes \phi)=0 \quad \text { and } \quad \phi \mu_{A}(\phi \otimes \phi)=0
$$

are proved in an analogous way to (16).

Next, we give an example of a commutative simplicial group $G$ where the homotopy operator of the Eilenberg-Zilber contraction associated to $G \times G$ is not an algebra homotopy.

Example 3.4. Let us consider

$$
E Z_{G, G}:\left\{C_{N}(G \times G), C_{N}(G) \otimes C_{N}(G), f, g, \phi\right\}
$$

where $G$ is the Eilenberg-Mac Lane complex of type $(\mathbf{Z}, 1)$, denoted by $K(\mathbf{Z}, 1)$, described in terms of the classifying complex $(W)$ as a commutative simplicial group (see May, 1967, Theorem 23.2).

- By Theorem 3.3, (27) is an almost-full contraction.

- It is easy to check that $\phi \mu_{C_{N}(G \times G)}(1 \otimes \phi)[([a] \times[b]) \otimes([a] \times[b])] \neq 0$ (module degeneracies). 
By applying Proposition 3.1, we conclude that (27) is not a full algebra contraction.

Remark 3.5. In general, the Eilenberg-Zilber contraction associated to two simplicial groups is not a full algebra contraction.

Let prove now that the twisted Eilenberg-Zilber contraction associated to two simplicial groups is a semi-full algebra contraction. We need some preliminary results which will be essential in the proof.

Let $(\alpha, \beta)$ be a $(p, q)$-shuffle. If $\alpha_{1}=0$, i.e., $(\alpha, \beta)=\left(0, \alpha_{2}, \ldots, \alpha_{p} ; \beta_{1}, \ldots, \beta_{q}\right)$, then $\bar{\alpha}$ denotes the set obtained from $\alpha$ by omitting $\alpha_{1}=0$, i.e., $\bar{\alpha}=\left(\alpha_{2}, \ldots, \alpha_{p}\right)$; in analogous way, $\bar{\beta}$ may be defined. The notation $\alpha-1$ defines the set obtained from $\alpha$ by subtracting 1 , i.e., $\alpha-1=\left(\alpha_{1}-1, \ldots, \alpha_{p}-1\right)$; the term $\beta-1$ is analogously defined.

Lemma 3.6 (May, 1967). Using the notations above:

1. Let $(\alpha, \beta)$ be a $(p, q)$-shuffle, with $\alpha_{1}=0$. Then, $(\bar{\alpha}-1, \beta-1)$ becomes $a(p-1, q)$ shuffle and $\operatorname{sg}(\bar{\alpha}-1, \beta-1)=\operatorname{sg}(\alpha, \beta)$. Furthermore, every $(p-1, q)$-shuffle may be constructed in this way.

2. Let $(\alpha, \beta)$ be a $(p, q)$-shuffle, with $\beta_{1}=0$. Then, $(\alpha-1, \bar{\beta}-1)$ is a $(p, q-1)$-shuffle and $\operatorname{sg}(\alpha-1, \bar{\beta}-1)=\operatorname{sg}(\alpha, \beta)+p$. Furthermore, every $(p, q-1)$-shuffle may be constructed in this way.

Proposition 3.7. Let $(G, \cdot),\left(G^{\prime}, \cdot\right)$ and $G^{\prime \prime}$ be three simplicial groups, $G^{\prime \prime}$ operating on the left of $G$ via $*$, and $\tau: G^{\prime} \longrightarrow G^{\prime \prime}$ be a twisting function. The following conditions are equivalent:

- The twisted cartesian product of fibre $G$, base $G^{\prime}$, group $G^{\prime \prime}$ and twisting function $\tau$ is a simplicial group with regards to the group multiplication of the banal cartesian product, $G \times G^{\prime}$ (i.e., the trivial group multiplication).

- The identity

$$
\tau\left(a^{\prime} \cdot b^{\prime}\right) *\left(\partial_{0} a \cdot \partial_{0} b\right)=\left(\tau a^{\prime} * \partial_{0} a\right) \cdot\left(\tau b^{\prime} * \partial_{0} b\right)
$$

is verified, for all $\left(a, a^{\prime}\right)$ and $\left(b, b^{\prime}\right)$ in $G \times G^{\prime}$.

Proof. The only difference between the simplicial sets $G \times{ }_{\tau} G^{\prime}$ and $G \times G^{\prime}$ is the definition of the zero face operator. Hence, $G \times{ }_{\tau} G^{\prime}$ is a simplicial group if and only if the rule

$$
\partial_{0}\left(\left(a, a^{\prime}\right) \cdot\left(b, b^{\prime}\right)\right)=\partial_{0}\left(a, a^{\prime}\right) \cdot \partial_{0}\left(b, b^{\prime}\right)
$$

is satisfied.

Expanding the left term of the equation (29)

$$
\partial_{0}\left(\left(a, a^{\prime}\right) \cdot\left(b, b^{\prime}\right)\right)=\partial_{0}\left(a \cdot b, a^{\prime} \cdot b^{\prime}\right)=\left(\tau\left(a^{\prime} \cdot b^{\prime}\right) *\left(\partial_{0} a \cdot \partial_{0} b\right), \partial_{0} a^{\prime} \cdot \partial_{0} b^{\prime}\right) .
$$


The right term of (29)

$$
\partial_{0}\left(a, a^{\prime}\right) \cdot \partial_{0}\left(b, b^{\prime}\right)=\left(\left(\tau a^{\prime} * \partial_{0} a\right) \cdot\left(\tau b^{\prime} * \partial_{0} b\right), \partial_{0} a^{\prime} \cdot \partial_{0} b^{\prime}\right) .
$$

Hence, the identity (29) is verified if and only if

$$
\tau\left(a^{\prime} \cdot b^{\prime}\right) *\left(\partial_{0} a \cdot \partial_{0} b\right)=\left(\tau a^{\prime} * \partial_{0} a\right) \cdot\left(\tau b^{\prime} * \partial_{0} b\right) .
$$

Theorem 3.8. Let $(G, \cdot),\left(G^{\prime}, \cdot\right)$ and $G^{\prime \prime}$ be three simplicial groups, $G^{\prime \prime}$ operating on the left of $G$ and $\tau: G^{\prime} \longrightarrow G^{\prime \prime}$ be a twisting function such that the twisted cartesian product of fibre $G$, base $G^{\prime}$, group $G^{\prime \prime}$ and twisting function $\tau$ constitutes a simplicial group with regards to the trivial group multiplication. Then, the morphism

$$
\delta: C_{N}\left(G \times G^{\prime}\right) \longrightarrow C_{N}\left(G \times G^{\prime}\right)\left(a, a^{\prime}\right) \longrightarrow \delta\left(a, a^{\prime}\right)=\left(\tau a^{\prime} * \partial_{0} a, \partial_{0} a^{\prime}\right)-\left(\partial_{0} a, \partial_{0} a^{\prime}\right)
$$

is an algebra perturbation datum for the Eilenberg-Zilber contraction

$$
E Z_{G, G^{\prime}}:\left\{C_{N}\left(G \times G^{\prime}\right), C_{N}(G) \otimes C_{N}\left(G^{\prime}\right), A W_{G, G^{\prime}}, E M L_{G, G^{\prime}}, S H I_{G, G^{\prime}}\right\} .
$$

Proof. Theorem 2.2 ensures that $\delta$ is a perturbation datum for $E Z_{G, G^{\prime}}$. So it remains to prove that $\delta$ is a derivation; that is,

$$
\delta \mu_{A}=\mu_{A}(1 \otimes \delta+\delta \otimes 1),
$$

where $\mu_{A}$ denotes the Eilenberg-Mac Lane product on $C_{N}\left(G \times G^{\prime}\right)$.

For the remainder of the proof $\left(a, a^{\prime}\right)$ and $\left(b, b^{\prime}\right)$ denote an element in $\left(G \times G^{\prime}\right)_{p}$ and in $\left(G \times G^{\prime}\right)_{q}$, respectively.

On one hand,

$$
\delta \mu_{A}\left[\left(a, a^{\prime}\right) \otimes\left(b, b^{\prime}\right)\right]=\delta\left(\sum_{(\alpha, \beta)}(-1)^{s g(\alpha, \beta)}\left(s_{\beta} a \cdot s_{\alpha} b, s_{\beta} a^{\prime} \cdot s_{\alpha} b^{\prime}\right)\right)
$$

the sum being taken over all $(p, q)$-shuffles $(\alpha, \beta)$

$$
\begin{aligned}
= & \sum_{(\alpha, \beta)}(-1)^{s g(\alpha, \beta)}\left(\tau\left(s_{\beta} a^{\prime} \cdot s_{\alpha} b^{\prime}\right) * \partial_{0}\left(s_{\beta} a \cdot s_{\alpha} b\right), \partial_{0}\left(s_{\beta} a^{\prime} \cdot s_{\alpha} b^{\prime}\right)\right) \\
& -\sum_{(\alpha, \beta)}(-1)^{s g(\alpha, \beta)}\left(\partial_{0}\left(s_{\beta} a \cdot s_{\alpha} b\right), \partial_{0}\left(s_{\beta} a^{\prime} \cdot s_{\alpha} b^{\prime}\right)\right)
\end{aligned}
$$

according to (28)

$$
\begin{aligned}
& =\sum_{(\alpha, \beta)}(-1)^{s g(\alpha, \beta)}\left(\left(\tau s_{\beta} a^{\prime} * \partial_{0} s_{\beta} a\right) \cdot\left(\tau s_{\alpha} b^{\prime} * \partial_{0} s_{\alpha} b\right), \partial_{0} s_{\beta} a^{\prime} \cdot \partial_{0} s_{\alpha} b^{\prime}\right) \\
& -\sum_{(\alpha, \beta)}(-1)^{s g(\alpha, \beta)}\left(\partial_{0} s_{\beta} a \cdot \partial_{0} s_{\alpha} b, \partial_{0} s_{\beta} a^{\prime} \cdot \partial_{0} s_{\alpha} b^{\prime}\right) \\
& =\sum_{\substack{(\alpha, \beta) \\
\beta_{1}=0}}(-1)^{s g(\alpha, \beta)}\left(s_{\bar{\beta}-1} a \cdot\left(s_{\alpha-1} \tau b^{\prime} * s_{\alpha-1} \partial_{0} b\right), s_{\bar{\beta}-1} a^{\prime} \cdot s_{\alpha-1} \partial_{0} b^{\prime}\right)
\end{aligned}
$$




$$
\begin{aligned}
& -\sum_{\substack{(\alpha, \beta) \\
\beta_{1}=0}}(-1)^{s g(\alpha, \beta)}\left(s_{\bar{\beta}-1} a \cdot s_{\alpha-1} \partial_{0} b, s_{\bar{\beta}-1} a^{\prime} \cdot s_{\alpha-1} \partial_{0} b^{\prime}\right) \\
& +\sum_{\substack{(\alpha, \beta) \\
\alpha_{1}=0}}(-1)^{s g(\alpha, \beta)}\left(\left(s_{\beta-1} \tau a^{\prime} * s_{\beta-1} \partial_{0} a\right) \cdot s_{\bar{\alpha}-1} b, s_{\beta-1} \partial_{0} a^{\prime} \cdot s_{\bar{\alpha}-1} b^{\prime}\right) \\
& -\sum_{\substack{\alpha, \beta) \\
\alpha_{1}=0}}(-1)^{s g(\alpha, \beta)}\left(s_{\beta-1} \partial_{0} a \cdot s_{\bar{\alpha}-1} b, s_{\beta-1} \partial_{0} a^{\prime} \cdot s_{\bar{\alpha}-1} b^{\prime}\right) \\
&
\end{aligned}
$$

On the other hand,

$$
\begin{aligned}
& \mu_{A}(1 \otimes \delta+\delta \otimes 1)\left(\left(a, a^{\prime}\right) \otimes\left(b, b^{\prime}\right)\right) \\
&= \mu_{A}\left((-1)^{\left|\left(a, a^{\prime}\right)\right|}\left(a, a^{\prime}\right) \otimes\left[\left(\tau b^{\prime} * \partial_{0} b, \partial_{0} b^{\prime}\right)-\left(\partial_{0} b, \partial_{0} b^{\prime}\right)\right]\right) \\
&+\mu_{A}\left(\left[\left(\tau a^{\prime} * \partial_{0} a, \partial_{0} a^{\prime}\right)-\left(\partial_{0} a, \partial_{0} a^{\prime}\right)\right] \otimes\left(b, b^{\prime}\right)\right) \\
&= \mu_{A}\left[(-1)^{p}\left(a, a^{\prime}\right) \otimes\left(\tau b^{\prime} * \partial_{0} b, \partial_{0} b^{\prime}\right)\right]-\mu_{A}\left[(-1)^{p}\left(a, a^{\prime}\right) \otimes\left(\partial_{0} b, \partial_{0} b^{\prime}\right)\right] \\
&\left.+\mu_{A}\left[\left(\tau a^{\prime} * \partial_{0} a, \partial_{0} a^{\prime}\right)\right] \otimes\left(b, b^{\prime}\right)\right)-\mu_{A}\left[\left(\partial_{0} a, \partial_{0} a^{\prime}\right) \otimes\left(b, b^{\prime}\right)\right] \\
&= \sum_{(\alpha, \hat{\beta})}(-1)^{p+s g(\alpha, \hat{\beta})}\left(s_{\hat{\beta}} a \cdot\left(s_{\alpha} \tau b^{\prime} * s_{\alpha} \partial_{0} b\right), s_{\hat{\beta}} a^{\prime} \cdot s_{\alpha} \partial_{0} b^{\prime}\right) \\
&-\sum_{(\alpha, \hat{\beta})}(-1)^{p+s g(\alpha, \hat{\beta})}\left(s_{\hat{\beta}} a \cdot s_{\alpha} \partial_{0} b, s_{\hat{\beta}} a^{\prime} \cdot s_{\alpha} \partial_{0} b^{\prime}\right) \\
&+\sum_{(\hat{\alpha}, \beta)}(-1)^{s g(\hat{\alpha}, \beta)}\left(\left(s_{\beta} \tau a^{\prime} * s_{\beta} \partial_{0} a\right) \cdot s_{\hat{\alpha}} b, s_{\beta} \partial_{0} a^{\prime} \cdot s_{\hat{\alpha}} b^{\prime}\right) \\
&-\sum_{(\hat{\alpha}, \beta)}(-1)^{s g(\hat{\alpha}, \beta)}\left(s_{\beta} \partial_{0} a \cdot s_{\hat{\alpha}} b, s_{\beta} \partial_{0} a^{\prime} \cdot s_{\hat{\alpha}} b^{\prime}\right) .
\end{aligned}
$$

The sums being taken over all $(p, q-1)$-shuffles $(\alpha, \hat{\beta})$ and $(p-1, q)$-shuffles $(\hat{\alpha}, \beta)$, respectively.

By Lemma 3.6, (30) and (34), (31) and (35), (32) and (36), (33) and (37) coincide two against two.

Theorem 3.9. Let $G^{\prime \prime}$ be a simplicial group operating on the left of $G$ and $\tau: G^{\prime} \rightarrow$ $G^{\prime \prime}$ be a twisting function such that the TCP $G \times{ }_{\tau} G^{\prime}$ is a simplicial group with regards to the trivial group multiplication. Then, the contraction associated to $G \times{ }_{\tau} G^{\prime}$ by applying Theorem 2.2 is a semi-full algebra contraction.

\section{Proof.}

- Theorems 3.3 and 3.8 state that $E Z_{G, G^{\prime}}$ is an almost-full algebra contraction and $\delta$ is an algebra perturbation of this contraction, respectively.

- Theorem 2.2 states that $E Z_{G, G^{\prime}}$ contraction can be perturbed into $T E Z_{G, G^{\prime}}$ taking $\delta$ as perturbation datum.

Hence, using Theorem 3.2, we obtain the desired conclusion. 


\section{AN EXAMPLE}

In the next example we show how it is possible to take advantage of the multiplicative properties of the chain complexes in order to compute homological models for some special fiber bundles in an economical way. Concretely, we work with the TCP induced from $K\left(\mathbf{Z}_{2}, 1\right) \times{ }_{\tau} K\left(\mathbf{Z}_{2}, 2\right)$ by the group endomorphism $f: \mathbf{Z} \rightarrow \mathbf{Z}_{\mathbf{2}}$.

In the sequel we follow the iterative simplicial description of $K(\pi, n)$ in terms of the classifying complex $(W)$ given in May (1967, Theorem 23.2).

Given

$$
\begin{aligned}
K\left(\mathbf{Z}_{\mathbf{2}}, 2\right) & \stackrel{\tau}{\longrightarrow} K\left(\mathbf{Z}_{\mathbf{2}}, 1\right) \\
\left(\left(g_{n-2}^{n-1}, \ldots, g_{0}^{n-1}\right), \ldots,\left(g_{0}^{1}\right),[]\right) & \longrightarrow\left(g_{n-2}^{n-1}, \ldots, g_{0}^{n-1}\right), \quad g_{i}^{j} \in \mathbf{Z}_{\mathbf{2}}
\end{aligned}
$$

the universal (principal) TCP $K\left(\mathbf{Z}_{\mathbf{2}}, 1\right) \times_{\tau} K\left(\mathbf{Z}_{\mathbf{2}}, 2\right)$ is defined. Now, making use of the (natural) extended simplicial group homomorphism $f: K(\mathbf{Z}, 2) \rightarrow K\left(\mathbf{Z}_{\mathbf{2}}, 2\right)$ from the group homomorphism $f: \mathbf{Z} \rightarrow \mathbf{Z}_{\mathbf{2}}$, we can consider the principal TCP

$$
E=K\left(\mathbf{Z}_{\mathbf{2}}, 1\right) \times_{\tau f} K(\mathbf{Z}, 2)
$$

induced from $K\left(\mathbf{Z}_{\mathbf{2}}, 1\right) \times{ }_{\tau} K\left(\mathbf{Z}_{\mathbf{2}}, 2\right)$ by $f$. It is a simple matter to prove that $E$ is a simplicial group. Hence, by Theorem 3.9,

$$
C_{N}\left(K\left(\mathbf{Z}_{2}, 1\right)\right) \otimes_{t} C_{N}(K(\mathbf{Z}, 2))
$$

is a DGA-algebra with the same product as that of $C_{N}\left(K\left(\mathbf{Z}_{2}, 1\right)\right) \otimes C_{N}(K(\mathbf{Z}, 2))$. Thus, $t \cap$ is a derivation.

On the other hand, combining the results of Eilenberg and Mac Lane (1953, 1954) and Real (2000) the following semi-full algebra contractions can be established

$$
C_{N}\left(K\left(\mathbf{Z}_{2}, 1\right)\right) \Rightarrow E\left(u_{1}, 1\right) \otimes \Gamma\left(v_{1}, 2\right)
$$

where $d\left(\gamma_{j}\left(v_{1}\right)\right)=2 u_{1} \gamma_{j-1}\left(v_{1}\right)$. (The last one is, actually, almost-full).

And

$$
C_{N}(K(\mathbf{Z}, 2)) \Rightarrow \Gamma\left(v_{2}, 2\right)
$$

Here $E(u, 1)$ denotes the exterior algebra with one generator $u$ in degree 1 and $\Gamma(v, 2)$ denotes the divided power algebra with one generator $v$ in degree 2 .

With this information at hand, it is immediate to establish

$$
C_{N}\left(K\left(\mathbf{Z}_{2}, 1\right)\right) \otimes C_{N}(K(\mathbf{Z}, 2)) \Rightarrow\left(E\left(u_{1}, 1\right) \otimes \Gamma\left(v_{1}, 2\right)\right) \otimes \Gamma\left(v_{2}, 2\right)
$$

This contraction can be perturbed into

$$
C_{N}\left(K\left(\mathbf{Z}_{2}, 1\right)\right) \otimes_{t} C_{N}(K(\mathbf{Z}, 2)) \Rightarrow\left(\left(E\left(u_{1}, 1\right) \otimes \Gamma\left(v_{1}, 2\right)\right) \otimes \Gamma\left(v_{2}, 2\right), d^{\otimes}+d_{t \cap}\right)
$$


taking $t \cap$ as the perturbation datum. The pointwise nilpotency condition is guaranteed using the standard filtration on $C_{N}\left(K\left(\mathbf{Z}_{\mathbf{2}}, 1\right)\right) \otimes C_{N}(K(\mathbf{Z}, 2))$ since $C_{N}\left(K\left(\mathbf{Z}_{2}, 1\right)\right)$ is reduced.

Let us observe that (38) is a semi-full algebra contraction and $t \cap$ is an algebra perturbation of this contraction. Then, by Theorem 3.2, the contraction (39) is semifull, hence $d_{t \cap}$ is a derivation. In particular, this mean that the right hand of (39) is a DGA-algebra. This fact has important consequences in order to compute $d_{t \cap}$ on the whole set of additive generators of the homological model (as a module). By BPL, we have

$$
d_{t \cap}=f(t \cap) \Sigma_{c}^{t \cap} g
$$

by direct computation we obtain

$$
d_{t \cap}\left(\gamma_{1}\left(v_{2}\right)\right)=u_{1}
$$

Taking into account that

$$
\gamma_{i}\left(v_{2}\right) \gamma_{j}\left(v_{2}\right)=\left(\begin{array}{c}
i+j \\
i
\end{array}\right) \gamma_{i+j}\left(v_{2}\right)
$$

and $d_{t \cap}$ is a derivation, we have

$$
d_{t \cap}\left(\gamma_{j}\left(v_{2}\right)\right)=u_{1} \gamma_{j-1}\left(v_{2}\right)
$$

By a similar argument we can calculate $d_{t \cap}$ on any additive generator of the module; in this way, it is easy to see that $d_{t \cap}$ is null on all additive generators except on $\gamma_{j}\left(v_{2}\right)$.

Summing up, in order to compute $d_{t \cap}$ over all additive generators of the module, we only use the formula (40) over the generators of the algebra: $u_{1}, v_{1}$, and $v_{2}$; for the remaining additive generators we use the multiplicative property of $d_{t \cap}$. Let us observe that this represents an economical alternative way to the formula (40) for computing $d_{t \cap}$.

\section{ACKNOWLEDGMENT}

The authors would like to thank the referee for their helpful suggestions. This work was partially supported by the PAICYT research project FQM-296 from Junta de Andalucía (Spain).

\section{REFERENCES}

Brown, E. H. (1959). Twisted tensor products. Ann. Math. 69:223-242.

Brown, R. (1967). The twisted Eilenberg-Zilber theorem. Celebrazioni Archimedee del secolo XX. Simposio di topologia pp. 34-37.

Eilenberg, S., Mac Lane, S. (1953). On the groups $H(\pi, n)$, I. Ann. Math. 58:55-106.

Eilenberg, S., Mac Lane, S. (1954). On the groups $H(\pi, n)$, II. Ann. Math. 66:49-149.

Eilenberg, S., Zilber, J. A. (1953). On products of complexes. Am. J. Math. 75:200-204. 
Gugenheim, V. K. A. M., Lambe, L. A. (1989). Perturbation theory in differential homological algebra I. Illinois J. Math. 33(4):566-582.

Gugenheim, V. K. A. M., Lambe, L. A., Stasheff, J. D. (1991). Perturbation theory in differential homological algebra II. Illinois J. Math. 35(3):357-373.

Huebschmann, J., Kadeishvili, T. (1991). Small models for chain algebras. Math. Z. 209:245-280.

Kadeishvili, T., Saneblidze, S. (2005). A cubical model for a fibration. J. Pure Appl. Algebra 196:203-228.

Lambe, L. A., Stasheff, J. D. (1987). Applications of perturbation theory to iterated fibrations. Manuscripta Math. 58:367-376.

Mac Lane, S. (1995). Homology. Classics in Mathematics. Berlin: Springer-Verlag. Reprint of the 1975 edition.

May, J. P. (1967). Simplicial Objects in Algebraic Topology. Princenton: Van Nostrad.

Real, P. (2000). Homological perturbation theory and associativity. Homology, Homotopy, and Applications 2:51-88.

Shih, W. (1962). Homologie des espaces fibrés. Inst. Hautes Etudes Sci. 13:93-176.

Szcsarba, R. H. (1961). Homology of twisted cartesian products. Trans. Amer. Math. Soc. 100:197-216. 